\title{
On the Dirichlet Eta Function
}

\section{Azkour $\mathbf{M}^{\star}$}

Ministry of National Education, Vocational Training, Higher Education and Scientific Research, Morocco

\begin{abstract}
This paper gives a proof of the following result: if $\eta(\rho)=0$ and $\Re(\rho)>0$, then:

$\lim _{k \rightarrow \infty} \frac{\mathcal{X}_{k}(\rho)}{\eta_{k}(\rho)}=2$.

$\eta$ is the Dirichlet eta function defined for $\Re(\mathrm{s})>0$ by $\eta(s)=\sum_{n=1}^{\infty} \mathcal{X}_{n}(s)$ with $X_{n}(s)=(-1)^{n-1} n^{-s}$ and $\eta_{k}(s)=\sum_{n=1}^{k} \mathcal{X}_{n}(s)$.
\end{abstract}

Keywords: Dirichlet eta function; Riemann zeta function; Complex variable

\section{Introduction}

We know that the Riemann zeta function $\zeta$ is the analytic function of the complex variable $s$, defined in the half-plane $\mathfrak{R}(s)>1$ by Sarnak [1]

$$
\zeta(s)=\sum n^{-s}=\prod\left(1-p^{-s}\right)^{-1}
$$

where the series $\sum n^{-s}$ is absolutely convergent for $\mathfrak{R}(s)>1$ and the product $\Pi\left(1-p^{-s}\right)^{-1}$ extends over all the prime numbers $p \in P=\{2,3,5,7, \ldots\}$ and as shown by Riemann, $\zeta$ can be continued analytically to $\mathbb{C} \backslash\{1\}$ as a meromorphic function and has a simple pole at $s=1$ with residue 1 [2]. We also know that $\zeta$ is defined for any complex number $s \neq 1$ and having $\mathfrak{R}(s)>0$ by

$$
\zeta(s)=\left(1-2^{1-s}\right)^{-1} \eta(s)
$$

where $\eta$ is the Dirichlet eta function which is defined in the half-plane $\mathfrak{R}(\mathrm{s})>0$ by [3]

$$
\eta(s)=\sum(-1)^{n-1} n^{-s}
$$

so, noticing that $\forall s \in \mathbb{C}:\left(1-2^{1-s}\right)^{-1} \neq 0$, we deduce that [4]:

"if $\zeta(s)=0$ and $\mathfrak{R}(s)>0$, then $\eta(s)=0$ ".

On the other hand, we know that $\zeta(s)$ is related to $\zeta(1-s)$ by the Riemann functional equation [5]

$$
\wedge(s)=\wedge(1-s)
$$

where $\Lambda(s)=\pi^{-s / 2} \Gamma(s / 2) \zeta(s)$ and $\Gamma$ is the Euler gamma function. So, by the two in eqns. (2) and (4), we obtain:

$$
" \forall 0<\Re(s)<1: \frac{\eta(1-s)}{\eta(s)}=\frac{f(s)}{f(1-s)} ; f(s)=\pi^{-s / 2} \Gamma(s / 2)\left(1-2^{s}\right) "
$$

And we have $\Gamma(s)$ does not vanish for any $s$ in $\mathbb{C}$ and has an infinity of simple poles with residue $(-1)^{n} / n$ ! at $s=-n$ where $n=0,1,2,3, \ldots$ etc.,

So

$$
\lim _{s \rightarrow \rho} \frac{f(s)}{f(1-s)}=0 \Rightarrow \rho=3,5,7,9 \ldots
$$

And

$\lim _{s \rightarrow \rho} \frac{f(s)}{f(1-s)}=\tilde{\infty} \Rightarrow \ldots,-8,-6,-4,-2=\rho$

Then

$$
" \forall 0<\mathfrak{R}(\rho)<1: \lim _{s \rightarrow \rho} \frac{f(s)}{f(1-s)} \neq 0, \tilde{\infty}
$$

that is to say for every complex number $\rho$ with $0<\mathfrak{R}(\rho)<1$, we have:

$$
\lim _{s \rightarrow \rho} \frac{\eta(s)}{\eta(1-s)} \neq 0, \tilde{\infty}
$$

this means that: "if $\eta(\rho)=0$ and $0<\mathfrak{R}(\rho)<1$, then $\eta(1-\rho)=0$ ".

So, for $\mathfrak{R}(\mathrm{s})>0$, we have:

$$
\eta(s)=\sum_{n \geq 1} \mathcal{X}_{n}(s) \text { with } \mathcal{X}_{n}(s)=(-1)^{n-1} n^{-s}
$$

let's denote:

$$
\eta_{k}(s)=\sum_{n=1}^{k} \mathcal{X}_{n}(s) \text { for } k \geq 1,
$$

if $\eta(\rho)=0$ and $\lim _{k \rightarrow \infty} \frac{\mathcal{X}_{n}(\rho)}{\eta_{k}(\rho)}=\tilde{\infty}$ then,

$$
\lim _{k \rightarrow \infty} \frac{\mathcal{X} k+1(\rho)}{\eta k+1(\rho)}=\tilde{\infty}
$$

and knowing that $\lim _{k \rightarrow \infty} \frac{\mathcal{X} k+1(\rho)}{\mathcal{X} k(\rho)}=\lim _{k \rightarrow \infty}(-1)\left(\frac{k}{k+1}\right)^{\rho}=-1$ we have

$$
\lim _{k \rightarrow \infty} \frac{\mathcal{X} k+1(\rho)}{\eta k+1(\rho)}=\lim _{k \rightarrow \infty} \frac{\frac{\mathcal{X} k+1(\rho)}{\mathcal{X} k(\rho)}}{\frac{\eta k+1(\rho)}{\mathcal{X} k(\rho)}}=\lim _{k \rightarrow \infty}=\frac{\frac{\mathcal{X} k+1(\rho)}{\mathcal{X} k(\rho)}}{\frac{\eta k(\rho)}{\mathcal{X} k(\rho)}+\frac{\mathcal{X} k+1(\rho)}{\mathcal{X} k(\rho)}}=\frac{-1}{\frac{1}{\tilde{\infty}}-1}=\tilde{\infty},
$$

this implies $1=\tilde{\infty}$, and this result is absurd, so if $\eta(\rho)=0$, then

$$
\lim _{k \rightarrow \infty} \frac{\mathcal{X} k(\rho)}{\eta k(\rho)} \neq \tilde{\infty},
$$

*Corresponding author: Azkour M, Ministry of National Education, Vocational Training, Higher Education and Scientific Research, Morocco, Tel: +21671766462; E-mail: mazkour@yahoo.com

Received September 18, 2018; Accepted October 03, 2018; Published October 10, 2018

Citation: Azkour M (2018) On the Dirichlet Eta Function. J Appl Computat Math 7 420. doi: 10.4172/2168-9679.1000420

Copyright: () 2018 Azkour M. This is an open-access article distributed under the terms of the Creative Commons Attribution License, which permits unrestricted use, distribution, and reproduction in any medium, provided the original author and source are credited. 
if $\eta(\rho)=0$ and $\lim _{k \rightarrow \infty} \frac{\mathcal{X}_{k}(\rho)}{\eta_{k}(\rho)}=\lambda \in \mathbb{C} \backslash\{0\}$, then

$$
\lim _{k \rightarrow \infty} \frac{\mathcal{X}_{k}+1(\rho)}{\eta_{k}+1(\rho)}=\lim _{k \rightarrow \infty} \frac{\frac{\mathcal{X}_{k}+1(\rho)}{\mathcal{X}_{k}(\rho)}}{\frac{\eta_{k}+1(\rho)}{\mathcal{X}_{k}(\rho)}}=\lim _{k \rightarrow \infty} \frac{\frac{\mathcal{X}_{k}+1(\rho)}{\mathcal{X}_{k}(\rho)}}{\frac{\eta_{k}(\rho)}{\mathcal{X}_{k}(\rho)}+\frac{\mathcal{X}_{k}+1(\rho)}{\mathcal{X}_{k}(\rho)}}=\frac{-1}{\frac{1}{\lambda}-1}=\lambda,
$$

that is to say

$$
\lambda=2 \text {, }
$$

if $\eta(\rho)=0$ and $0<\mathfrak{R}(\rho)<1$, then

$$
\lim _{k \rightarrow \infty} \frac{\mathcal{X}_{\mathrm{k}}(\rho)}{\eta_{k}(\rho)}=0,2, \nexists \text { and } \lim _{k \rightarrow \infty} \frac{\mathcal{X}_{k}(1-\rho)}{\eta_{k}(\rho)}=0,2, \nexists\left(e \cdot g \lim _{k \rightarrow \infty} e^{i k}=\nexists\right)
$$

and

$$
\lim _{k \rightarrow \infty} \frac{\frac{\mathcal{X}_{k}(\rho)}{\eta_{k}(\rho)}}{\mathcal{X}_{k}(1-\rho)}=\frac{0,2, \nexists}{0,2, \nexists}=0,1, \gamma \neq 1, \tilde{\infty}, \nexists=\lim _{k \rightarrow \infty} \frac{\eta_{k}(1-\rho)}{\eta_{k}(\rho)} \frac{\mathcal{X}_{k}(\rho)}{\mathcal{X}_{k}(1-\rho)}
$$

for $0<\Re(\rho)<1: \lim _{k \rightarrow \infty} \frac{\mathcal{X}_{k}(\rho)}{\mathcal{X}_{k}(1-\rho)}=0, \tilde{\infty}, \nexists$

$$
\begin{aligned}
& \text { if } \lim _{k \rightarrow \infty} \frac{\mathcal{X}_{k}(\rho)}{\mathcal{X}_{k}(1-\rho)}=0 \text { and } \lim _{k \rightarrow \infty} \frac{\eta_{k}(1-\rho)}{\eta_{k}(\rho)} \neq \tilde{\infty}, \text { then } \\
& \lim _{k \rightarrow \infty} \frac{\eta_{k}(1-\rho)}{\eta_{k}(\rho)} \frac{\mathcal{X}_{k}(\rho)}{\mathcal{X}_{k}(1-\rho)}=0=\frac{0}{0,2, \nexists}
\end{aligned}
$$

that is to say if $\eta(\rho)=0$ and $\frac{1}{2}<\mathfrak{R}(\rho)<1$ and $\lim _{k \rightarrow \infty} \frac{\eta_{k}(1-\rho)}{\eta_{k}(\rho)} \neq \tilde{\infty}$

then

$$
\lim _{k \rightarrow \infty} \frac{\mathcal{X}_{k}(\rho)}{\eta_{k}(1-\rho)}=0
$$

but, it is very likely that if $\eta(\rho)=0$, then

$$
\lim _{k \rightarrow \infty} \frac{\mathcal{X}_{k}(\rho)}{\eta_{k}(\rho)}=2 \neq 0
$$

let $f_{\mathrm{n}, \mathrm{a}}$ be the function of the variable $x$ defined by

$$
f_{n, \alpha}(x)=1-\left(\frac{x}{x+1}\right)^{\alpha}+\left(\frac{x}{x+2}\right)^{\alpha}-\left(\frac{x}{x+3}\right)^{\alpha}+\ldots+(-1)^{n}\left(\frac{x}{x+n}\right)^{\alpha}
$$

Then

$$
\lim _{\substack{n \rightarrow \infty \\ n / x \rightarrow 0}} f_{n, \alpha}(x)=1-1+1-1+\ldots+(-1)^{n}=\frac{1}{2}(\text { Grandi'series })
$$

let's take:

$$
\eta_{m}(s)=\eta_{k}(s)+\eta_{k^{\prime} m}(s) \text { with } \eta_{k, m}(s)=\sum_{n=k+1}^{m} \mathcal{X}_{n}(s)
$$

so, if $\eta(\rho)=0$, then:

$$
\lim _{m \rightarrow \infty} \eta_{m}(\rho)=0 \text { and } \lim _{m \rightarrow \infty} \frac{\eta_{k, m}(\rho)}{\eta_{k}(\rho)}=-1
$$

and we have $\forall n \geq 0$ :

$$
\frac{\mathcal{X}_{\mathrm{k}+\mathrm{n}}(\rho)}{\mathcal{X}_{k}(\rho)}=(-1)^{n}\left(\frac{k}{k+n}\right)^{\rho}
$$

so, using $f_{\mathrm{n}, \mathrm{a}}$, we have:

$$
1+\sum_{n=1}^{m} \frac{\mathcal{X}_{k+n}(\rho)}{\mathcal{X}_{k}(\rho)}=1+\sum_{n=1}^{m}(-1)^{n}\left(\frac{k}{k+n}\right)^{\rho}=f_{m, \rho}(k)
$$

and

$$
\lim _{\substack{m \rightarrow \infty \\ \mathrm{m} / x \rightarrow 0}} f_{\mathrm{m}, \rho}(x)=1-1+1-1+\ldots+(-1)^{m}=\frac{1}{2}
$$

that is to say

$$
1+\lim _{\substack{m \rightarrow \infty \\ \mathrm{m} / x \rightarrow 0}} \sum_{n=1}^{m} \frac{\mathcal{X}_{k+n}(\rho)}{\mathcal{X}_{k}(\rho)}=1+\lim _{\substack{m \rightarrow \infty \\ \mathrm{m} / x \rightarrow 0}} \frac{\sum_{n=1}^{m} \mathcal{X}_{k+n}(\rho)}{\mathcal{X}_{k}(\rho)}=1+\lim _{\substack{m \rightarrow \infty \\ \mathrm{m} / x \rightarrow 0}} \frac{\eta_{k, m}(\rho)}{\mathcal{X}_{k}(\rho)}=\frac{1}{2}
$$

then

$$
1+\lim _{\substack{m \rightarrow \infty \\ \mathrm{m} / x \rightarrow 0}} \frac{\eta_{k, m}(\rho)}{\eta_{k}(\rho)} \frac{\eta_{k}(\rho)}{\mathcal{X}_{k}(\rho)}=1-\lim _{k \rightarrow \infty} \frac{\eta_{k}(\rho)}{\mathcal{X}_{k}(\rho)}=\frac{1}{2}
$$

that is to say

$$
\lim _{k \rightarrow \infty} \frac{\eta_{k}(\rho)}{\mathcal{X}_{k}(\rho)}=\frac{1}{2}
$$

So

$$
\lim _{k \rightarrow \infty} \frac{\mathcal{X}_{k}(\rho)}{\eta_{k}(\rho)}=2 .
$$

\section{References}

1. Sarnak P (2004) Problems of the Millennium: The Riemann Hypothesis Princeton University and Courant Institute of Math. Sciences.

2. Borwein P, Choi S, Rooney B, Weirathmueller A (2007) The Riemann Hypothesis: A Resource for the Afficionado and Virtuoso Alike. Springer, Berlin, Heidelberg NewYork, Hong Kong, London, Milan Paris, Tokyo.

3. Jaiswal AK, Khandelwal A (2009) A text book of computer based numerical and statistical techniques, p: 618

4. Iyengar SRK, Jain RK (2009) Numerical Methods, New Age International Publishers, pp: 182-184.

5. Zaliznaik V (2008) Essentials of scientific computing (1stedn.), Numerical Methods in Science and Engineering. 